\title{
Gemcitabine and docetaxel in relapsed and unresectable high-grade osteosarcoma and spindle cell sarcoma of bone
}

\author{
E. Palmerini ${ }^{1,9^{*}}$, R. L. Jones ${ }^{2}$, E. Marchesi ${ }^{1}$, A. Paioli ${ }^{1}$, M. Cesari ${ }^{1}$, A. Longhi ${ }^{1}$, C. Meazza ${ }^{3}$, L. Coccoli ${ }^{4}$, F. Fagioli ${ }^{5}$, \\ S. Asaftei ${ }^{5}$, G. Grignani ${ }^{6}$, A. Tamburini ${ }^{7}$, S. M. Pollack ${ }^{2}$, P. Picci ${ }^{8}$ and S. Ferrari ${ }^{1}$
}

\begin{abstract}
Background: Few new compounds are available for relapsed osteosarcoma. We retrospectively evaluated the activity of gemcitabine $(\mathrm{G})$ plus docetaxel $(\mathrm{D})$ in patients with relapsed high-grade osteosarcoma and high-grade spindle cell sarcoma of bone (HGS).

Methods: Patients receiving G $900 \mathrm{mg} / \mathrm{m}^{2}$ d 1, 8; D $75 \mathrm{mg} / \mathrm{m}^{2}$ d 8, every 21 days were eligible. Primary end-point: progression-free survival (PFS) at 4 months; secondary end-point: overall survival (OS) and response rate.

Results: Fifty-one patients were included, with a median age of 17 years (8-71), 26 (51\%) were pediatric patients. GD line of treatment: 2nd in 14 patients, $\geq 3$ rd in 37.25 (49\%) patients had metastases limited to lungs, 26 (51 \%) multiple sites. Histology: 40 (78 \%) osteosarcoma, 11 (22\%) HGS. Eight (16\%) patients achieved surgical complete response (sCR2) after GD.

Four-month PFS rate was $46 \%$, and significantly better for patients with ECOG 0 (ECOG 0: $54 \%$ vs ECOG 1: $43 \%$ vs ECOG 2: $0 \%$; $p=0.003$ ), for patients undergoing metastasectomy after GD (sCR2 $75 \%$ vs no-sCR2 $40 \%, p=0.02$ ) and for osteosarcoma (osteosarcoma $56 \%$ vs HGS $18 \% ; p=0.05$ ), with no differences according to age, line of treatment, and pattern of metastases.

Forty-six cases had RECIST measurable disease: 6 (13\%) patients had a partial response (PR), 20 (43\%) had stable disease (SD) and 20 (43\%) had progressive disease (PD).

The 1-year OS was $30 \%: 67 \%$ for PR, $54 \%$ for SD and $20 \%$ for PD ( $p=0.005)$.

Conclusions: GD is an active treatment for relapsed high-grade osteosarcoma, especially for ECOG 0 patients, and should be included in the therapeutic armamentarium of metastatic osteosarcoma.
\end{abstract}

Keywords: Osteosarcoma, High-grade bone sarcoma, Gemcitabine, Docetaxel, Chemotherapy

\section{Background}

At present, patients with nonmetastatic osteosarcoma of the extremity under the age of 40 years, have an expected 5-year survival rate of $70 \%$ with multi-modality management consisting of chemotherapy (based on methotrexate, cisplatin, doxorubicin and ifosfamide) and surgery $[1,2]$.

\footnotetext{
* Correspondence: emanuela.palmerini@ior.it

'PROMETEO Laboratory/Chemotherapy Unit, Istituto Ortopedico Rizzoli, Bologna, Italy

${ }^{9}$ PROMETEO Laboratory/Section of Chemotherapy, Research, Innovation \& Technology (RIT) Department, Istituto Ortopedico Rizzoli, Via Pupilli, 1, 40136 Bologna, Italy

Full list of author information is available at the end of the article
}

While the outcome of patients with localized osteosarcoma of bone has improved with the introduction of multi-agent chemotherapy in combination with surgery $[1,2]$, treatment options for patients with relapsed disease are limited and post-relapse survival is poor, with a 5-year post relapse survival (PR) rate below $30 \%$ [3].

The role of second-line chemotherapy for recurrent osteosarcoma is much less well defined, and there is no accepted standard regimen. Treatment choice may take into account the prior disease-free interval, and often includes ifosfamide \pm etoposide \pm carboplatin, and other active drugs [4]. 
High-dose ifosfamide (HDIFO) has been widely used for patients with metastatic osteosarcoma [5, 6], but, no new drugs were FDA or EMA approved over the last 25 years.

Prospective trials with agents such as pemetrexed or sorafenib and sorafenib/everolimus were performed [7-9]. Some of these agents have shown modest activity in osteosarcoma, but none were deemed worthy of further development.

In general, there are few indications for radiation therapy, but there are anatomical locations in which the possibility of complete surgical resection is limited. In these cases, radiation may be an option to try to extend the progression-free interval. Novel local treatment techniques (e.g. proton beam therapy, radiofrequency ablation and isolated limb perfusion) may have a role in specific patients, under the management of a multi disciplinary team [4].

The combination of gemcitabine (G) plus docetaxel (D) is active in soft tissue sarcomas, with published data indicating higher activity than gemcitabine alone [10-12]. Although the biology of soft tissue sarcomas is fundamentally different from that of bone sarcoma, the efficacy of these two drugs has also been investigated in patients with recurrent osteosarcoma with conflicting results (Table 1; [13-18]).

Here we report the results of a retrospective multicenter analysis of the activity of this combination in patients with recurrent high-grade osteosarcoma and high-grade spindle cell sarcoma of bone (HGS).

This analysis involves both pediatric and adult patients, primarily as management for patients with localized disease is the same, regardless of age.

\section{Methods}

A joint analysis between the Italian Sarcoma Group and the Sarcoma Center of the University of Washington was planned, in order to collect data on patients with metastatic high grade bone sarcomas treated with GD.

This study was approved by the ethics committees of all 6 centers participating to the study: 5 Italian referral centers (Rizzoli Institute, Bologna; Tumor National Institute, Milan; Pediatric Oncology Departments of
Turin; Meyer Children's Hospital, Florence; Pediatric Onco-Hematology, Pisa) and the Sarcoma Center of the University of Washington.

All patients included in the study signed informed consent for treatment and privacy according to each individual institution's requirements.

The analysis period was set from January 2012 and August 2014.

Patients with the following characteristics were included: 1) diagnosis of high-grade osteosarcoma and spindle cell sarcoma of bone, 2) recurrent or advanced disease not amenable of surgical treatment, 3) disease progression after at least one line of chemotherapy, 4) treatment with GD, 5) availability of demographic, clinical and follow-up data. The following were required for cases evaluable for response 1) treated with at least 2 cycles of GD, 2) having measurable disease as per RECIST 1.1 and 3) with radiological images for review.

The diagnosis was confirmed in all cases by an experienced bone sarcoma pathologist.

Drugs were administered as follows: G 675-900 mg/m² over $90 \mathrm{~min}$ on Day 1 and G $675-900 \mathrm{mg} / \mathrm{m}^{2}$ and D $75 \mathrm{mg} / \mathrm{m}^{2}$ on Day 8 . All patients received pre-medication with steroids prior to docetaxel.

Patient characteristics including age, gender, ECOG performance status, primary tumor site, site and number of metastatic lesions, type and number of prior treatments, response to therapy, toxicity, date of progression, date of last follow-up or death were obtained from the databases or the patient clinical chart and collected in a study-specific case report form.

All relevant radiological images we re-reviewed for the purpose of this study (EP, RLJ). Response was assessed using the Response Evaluation Criteria In Solid Tumors (RECIST) version 1.1. Patients were assessed for response after the first 2 cycles and, in case of response or stable disease, every 2 or 3 cycles of therapy. Objective response was expressed as response rate (complete response $[\mathrm{CR}]+$ partial response $[\mathrm{PR}]$ ), stable disease $(\mathrm{SD})$ or progressive disease (PD).

Toxicity data were collected from clinical chart and from "patient-toxicity" questionnaires, in some of the centers. Toxicity was graded according to the Common

Table 1 Gemcitabine and docetaxel in advanced osteosarcoma

\begin{tabular}{lllll}
\hline Drugs & Pts n. & RR & CR/PR & Authors \\
\hline G 1,000 mg/m2 & 7 & $0 \%$ & $0 / 0$ & Merimsky O, Sarcoma 2000 [13] \\
G 675 mg/m2 D 75-100 mg/m2 & 10 & $30 \%$ & $0 / 3$ & Navid F, Cancer 2008 [14] \\
G 900 mg/m2 D 80-100 mg/m2 & 14 & $7 \%$ & $0 / 1$ & Fox E. SARC 003, Oncologist 2010 [15] \\
G 675 mg/m2 \pm D 75-100 mg/m2 & 4 & $25 \%$ & $0 / 1$ & Gosiengfiao Y, J Pediatr Hematol Oncol 2012 [16] \\
G 675 mg/m2 + D 75-100 mg/m2 & 18 & $5 \%$ & $0 / 1$ & Qi WX, Jpn J Clin Oncol 2012 [17] \\
G 675-900 mg/m2 D 100 mg/m2 & 17 & $24 \%$ & $3 / 1$ & Song BS, Pediatr Blood Cancer 2014 [18] \\
\hline
\end{tabular}

$G D$ gemcitabine and docetaxel, $R R$ response rate, $C R$ complete response, $P R$ partial response 
Toxicity Criteria for Adverse Events (CTCAE) version 4. In case of grade 4 neutropenia prophylactic use of GCSF was allowed; therapeutic use of G-CSF was mandatory in case of febrile neutropenia.

Treatment was discontinued at progression or unacceptable toxicity. All patients who received at least one cycle (one cycle was defined as G on Day 1 and GD on Day 8, every 21 days) were included in an intentionto-treat analysis.

Progression-Free Survival (PFS) and Overall Survival (OS), were estimated according to the Kaplan and Meier method with their respective $95 \%$ confidence intervals (CI) and calculated from the first day of chemotherapy administration to tumor progression (PFS) or death or last follow-up visit (OS).

Metastasectomy was performed on a "case-by-case" basis, following multidisciplinary discussion. Only patients with confirmed response (partial response or stable disease after 2 consecutive assessments) were considered for surgical removal of metastases. If excision of all secondary lesions became possible, patients were classified as achieving a second surgical complete remission (sCR2).

\section{Results}

Fifty-one patients were included in the study. The clinical characteristics are shown in Table 2. Twenty-six patients $(51 \%)$ were aged less than 18 years. Most of the patients had an ECOG performance status of 0 and $73 \%$ had received 1 or more chemotherapy lines (with a maximum of 5 lines). 40 (78 \%) patients had high-grade osteosarcoma and 11 (22\%) had HGS. The median age was 17 years (range 8 to 71 years): 14.5 years (range 8 to 59) for osteosarcoma patients and 36 years (range 18 to 71) for the 11 patients with HGS.

All patients had received neoadjuvant/adjuvant chemotherapy with doxorubicin (cumulative dose 360$\left.420 \mathrm{mg} / \mathrm{m}^{2}\right)$, cisplatin $\left(600 \mathrm{mg} / \mathrm{m}^{2}\right)$, ifosfamide (30-60 $\left.\mathrm{gr} / \mathrm{m}^{2}\right)$, while methotrexate $\left(36-60 \mathrm{gr} / \mathrm{m}^{2}\right)$, was administered to all patients younger than 40 years.

Fourteen (27\%) patients received GD at their first recurrence. Thirty-seven patients received GD combination after failure of prior chemotherapy lines (with a maximum of 5 lines) (Table 2). HDIFO (ifosfamide $15 \mathrm{~g} / \mathrm{m}^{2}$ plus mesna as a 5 day-continuous infusion or $14 \mathrm{gr} / \mathrm{m}^{2}$ in 14 day-continuous infusion) was offered to all cases prior to GD: in 1st line, or in the adjuvant setting in case of poor response to neoadjuvant chemotherapy. Other drugs employed in the metastatic setting were: cyclophosphamide and etoposide, ifosfamide and etoposide, sorafenib, sorafenib and everolimus, pemetrexed, vinorelbine and anti IGF-1R based therapies.

The schedule of gemcitabine at a lower dose $\left(675 \mathrm{mg} / \mathrm{m}^{2}\right)$ was employed in 8 cases, mainly in pediatric patients.
Table 2 Clinical, Pathologic and Treatment Variable patients with osteosarcoma and high grade spindle cell sarcoma of bone

\begin{tabular}{|c|c|c|}
\hline Characteristics & Pts $n$. & $\%$ \\
\hline \multicolumn{3}{|l|}{ Histology } \\
\hline Osteosarcoma & 40 & $78 \%$ \\
\hline HGS & 11 & $22 \%$ \\
\hline \multicolumn{3}{|l|}{ Gender } \\
\hline Male & 36 & $70 \%$ \\
\hline Female & 15 & $30 \%$ \\
\hline \multicolumn{3}{|l|}{ Age } \\
\hline$\geq 18$ years & 25 & $49 \%$ \\
\hline$<18$ years & 26 & $51 \%$ \\
\hline \multicolumn{3}{|l|}{ ECOG } \\
\hline 0 & 33 & $64 \%$ \\
\hline 1 & 14 & $28 \%$ \\
\hline 2 & 4 & $8 \%$ \\
\hline \multicolumn{3}{|c|}{ Line of $\mathrm{CT}$ for metastases } \\
\hline 1 & 14 & $27 \%$ \\
\hline 2 & 28 & $55 \%$ \\
\hline$\geq 3$ & 9 & $18 \%$ \\
\hline \multicolumn{3}{|c|}{ Pattern of metastases } \\
\hline Lung Only & 25 & $49 \%$ \\
\hline Multiple & 26 & $51 \%$ \\
\hline \multicolumn{3}{|l|}{ Bone metastases } \\
\hline Yes & 13 & $25 \%$ \\
\hline No & 38 & $75 \%$ \\
\hline
\end{tabular}

HGS High grade spindle cell sarcoma of bone, GD gemcitabine and docetaxel, CT chemotherapy

\section{Response assessment}

Five patients were excluded from the response evaluation: two patients did not receive docetaxel due to an allergic reaction, one for grade 4 skin toxicity after the first cycle and 2 patients with metastatic lesions that were non measurable by RECIST.

In 46 patients evaluable for response: 6 patients achieved a PR (6/46 [13\%]) and 20 (43\%) SD, with a median duration of response lasting 6.5 months (range 2-11) for PRs and 4 months (range 2 to 16 months), for patients with SD. All patients achieving a partial response had classic osteosarcoma histology, and 5 of them were pediatric. Twenty out of 46 patients experienced progressive disease (PD) $(20 / 46,43 \%)$ (Fig. 1, Table 3).

\section{Toxicity}

The safety analysis is based on the 51 patients who received at least one dose of chemotherapy. The median number of cycles administered was 4 (range 1-20 cycles). Grade 4 hematological toxicity was observed in 13 


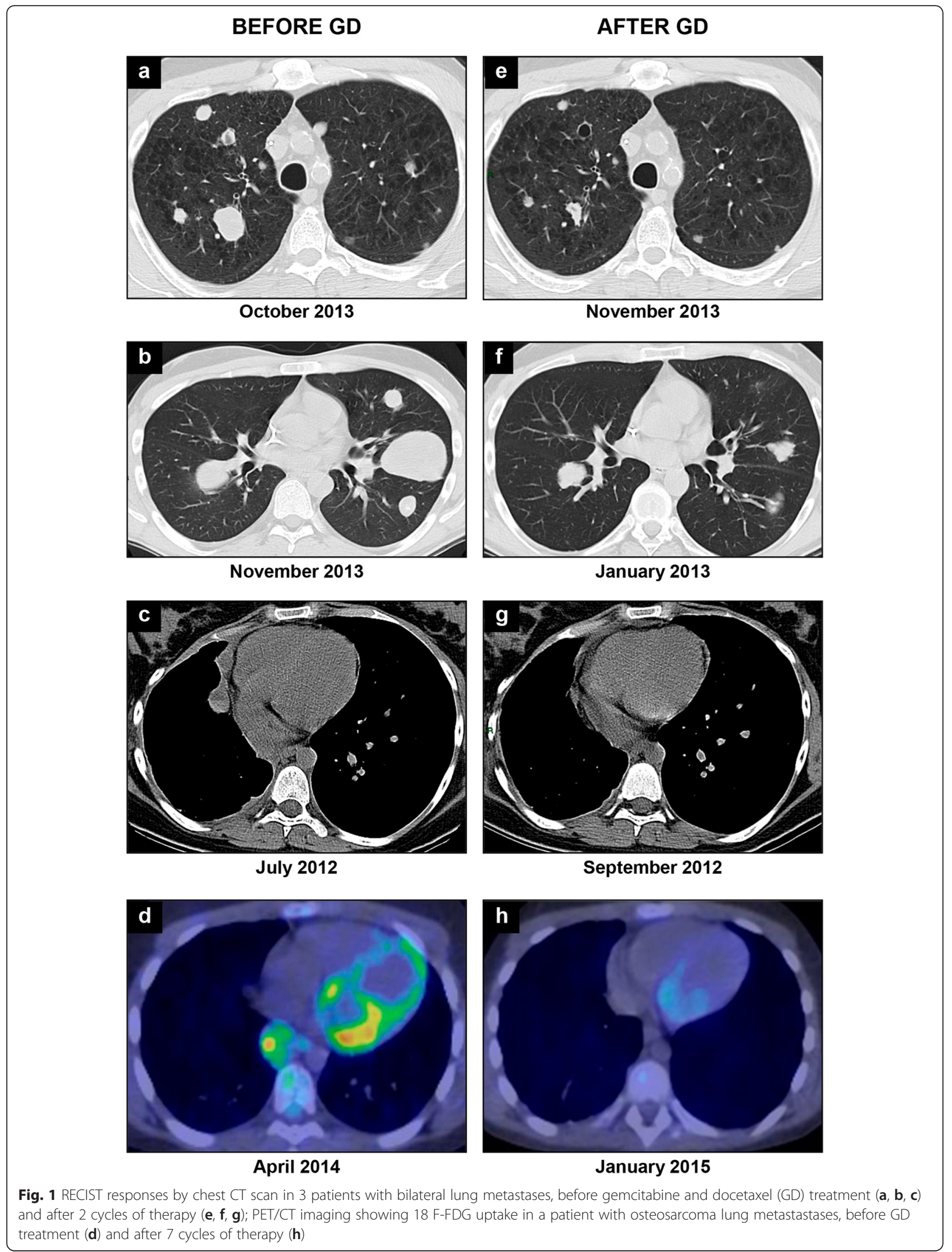


Table 3 Grade 3-4 non-hemathologic toxicity in 51 patient receiving gemcitabine and docetaxel (GD)

\begin{tabular}{llll}
\hline G3-4 non-hemathologic toxicity & Compliance & Outcome & GD cys \\
\hline Allergic reaction (1st cycle) & Stop docetaxel & Resolved & Resolved \\
Allergic reaction (1st cycle) & Stop docetaxel & Resolved & 4 \\
Allergic reaction (4th cycle) & Stop docetaxel & Resolved & 5 \\
Capillary leak syndrome & Stop docetaxel & Resolved & 9 \\
Diarrhea & Delay/dose reduction & Resolved & 6 \\
Febrile neutropenia & Delay & Resolved & 7 \\
Lung fibrosis & None & Resolved & 5 \\
Foot syndrome & None & Off -treatment & 2 \\
Stephen Johnson syndrome & Stop docetaxel & & 1 \\
\hline
\end{tabular}

GD cys gemcitabine and docetaxel number of chemoteherapy cycles

(25\%) patients, with 11 (21\%) experiencing grade 4 neutropenia and two grade 4 thrombocytopenia. Nonhematological toxicity was reported in 8 (16\%) patients, with $3(6 \%)$ patients experiencing allergic reactions (two following the first cycle and one after 4 cycles). Diarrhea (grade 1) was reported in 2 cases, lung fibrosis, Steven Johnson syndrome and capillary leak syndrome in one patient each (Table 4).

No differences of toxicity were observed according to G dose.

\section{Outcome}

\section{Progression free survival}

The median PFS was 3.5 months (range 1-13.5 months). The 4- and 6-month PFS were $46 \%$ (95\% CI; 31-61\%) and $28 \%$ (95\% CI, 15-42), respectively (Fig. 2a, Table 5). The 4-month PFS was significantly better for patients with osteosarcoma compared to HGS (Fig. 2b). No significant difference was observed according to age, gender, number of lines of systemic therapy and pattern of metastatic spread. Patients with a good performance status (ECOG 0) had significantly better PFS (Table 5).

\section{Overall survival post GD}

The median survival time post gemcitabine plus docetaxel was 7.5 months (range 2-45 months).

The 1-year overall survival was $30 \%$ (95\% CI; 22-57). For patients achieving a PR the 1-year OS was $67 \%$ (95\% CI; 13-100), for those with SD it was 54\% (95\% CI;

Table 4 Responses according to histology in 46 patients with measurable disease by RECIST

\begin{tabular}{llll}
\hline & Osteosarcoma $(n=35) n(\%)$ & HGS $(n=11) n(\%)$ & All (46) $n(\%)$ \\
\hline PR & $6(17)$ & 0 & $6(13)$ \\
SD & $14(40)$ & $6(55)$ & $20(43)$ \\
PD & $15(43)$ & $5(45)$ & $20(43)$
\end{tabular}

HGS High grade spindle cell sarcoma of bone
25-85), and for those with PD $20 \%$ (95\% CI; 1038) $(p=0.005)$.

Following GD, 8 patients underwent surgery and achieved a surgical complete response (sCR2). Six of these patients had lung metastases, 1 had a gastric lesion and another patient had a local recurrence with a synchronous subcutaneous metastasis. All these patients had osteosarcoma and the median age was 14.5 years (range 11-23 years). The 4-month PFS for patients achieving sCR2 was $75 \%$ (95\% CI; 45-100), and $49 \%$ (95\% CI; 24-56) for the others, $p=0.02$ (Fig. 3).

\section{Discussion}

Our multi-institutional retrospective study evaluated the activity of GD in a group of heavily pretreated patients with high grade osteosarcomas and HGS.

Our study demonstrated a 4-month PFS of $46 \%$, with better PFS in patients with a good performance status. Also, histology seems an important predictor of response, with a 4-month PFS of $56 \%$ in patients with classic high-grade osteosarcoma, as compared to a 4month PFS of only $18 \%$ for HGS. Of note, all pediatric patients had a diagnosis of classic osteosarcoma. These findings were confirmed in terms of response rate, with partial responses observed only in patients with highgrade osteosarcoma, whereas no objective response was seen in the HGS cohort, suggesting that patients with recurrent HGS should be offered participation in clinical trials of novel agents.

The PFS observed in the present series $(46 \%$ at 4 months) is superior to the 4-month PFS value of $40 \%$, the threshold to identify active agents in advanced soft tissue sarcomas set by Van Glabbeke and colleagues [19]. It is also superior to the PFS of a similar group of patients treated in a prospective Phase II study with pemetrexed (3-month PFS $17.2 \%$ (95 \% CI: 3.5-31) [7], and equivalent to that of sorafenib (4-month PFS $46 \%$ ) and sorafenib + everolimus combination (4-month PFS > $55 \%)[8,9]$. 

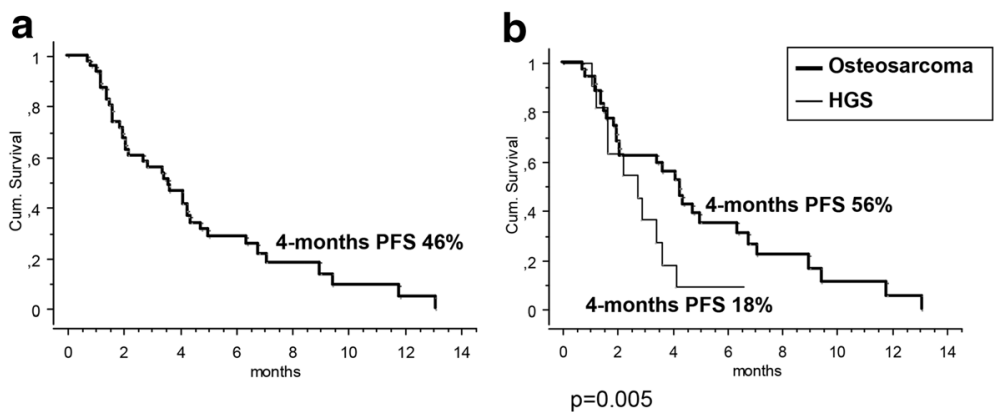

Fig. 2 Progression-free survival curves at 4 months (4-month PFS) in all 51 patients (a) and by histology (b): high-grade osteosarcoma (40 pts) and high-grade spindle cell sarcoma of bone (HGS; 11 pts)

The response rate for GD was $13 \%$ (17\% if we exclude HGS). With HDIFO, the treatment of choice in 1 st line for metastatic osteosarcoma, response rates described range between 10 and $62 \%[6,20,21]$, while for other drugs such as cyclophosphamide and etoposide, objective responses were seen in $19 \%$ and $28.5 \%$ of patients respectively in different studies $[22,23]$. With sorafenib, as monotherapy, or in combination with m-TOR

Table 5 Univariate Analysis of Clinical, Pathologic and Treatment Variable for PFS in patients with osteosarcoma and high grade spindle cell sarcoma of bone

\begin{tabular}{|c|c|c|c|c|}
\hline Characteristics & Pts n. & $\%$ 4-months PFS & $95 \% \mathrm{Cl}$ & $P$-value \\
\hline \multicolumn{5}{|l|}{ Overall } \\
\hline & 46 & 46 & $31-61$ & \\
\hline \multicolumn{5}{|l|}{ Age } \\
\hline$\geq 18$ years & 23 & 65 & $43-88$ & 0.4 \\
\hline$<18$ years & 23 & 35 & $17-53$ & \\
\hline \multicolumn{5}{|l|}{ Sex } \\
\hline Female & 12 & 50 & $22-78$ & 0.7 \\
\hline Male & 34 & 44 & $27-62$ & \\
\hline \multicolumn{5}{|l|}{ Mets Site } \\
\hline Lung only & 24 & 61 & $41-81$ & 0.1 \\
\hline Multiple & 22 & 30 & $11-50$ & \\
\hline \multicolumn{5}{|l|}{ Line GD } \\
\hline 2 & 13 & 60 & $32-87$ & 0.4 \\
\hline$\geq 3$ & 33 & 41 & 24.58 & \\
\hline \multicolumn{5}{|l|}{ ECOG } \\
\hline 0 & 29 & 54 & $35-72$ & 0.003 \\
\hline 1 & 13 & 43 & $15-71$ & \\
\hline 2 & 4 & 0 & 0 & \\
\hline \multicolumn{5}{|l|}{ Histology } \\
\hline Osteosarcoma & 35 & 56 & $39-73$ & 0.005 \\
\hline HGS & 11 & 18 & $0-41$ & \\
\hline
\end{tabular}

PFS Progression Free Survival, HGS high grade spindle cell sarcoma of bone, $G D$ gemcitabine and docetaxel inhibitors, the response rate is very low (sorafenib: $8 \%$, sorafenib + everolimus: $5 \%$ ) $[8,9]$.

There are few published data assessing the activity of GD in patients with recurrent osteosarcoma. Conflicting results are reported and most of the studies were based on a small number of patients (Table 1; [13-18]).

In a study by Song et al., a response rate of $24 \%$ was described in 17 pediatric patients (3 CR and $1 \mathrm{PR}$ ) [18]. The median OS was 9 months (range, 0.6-79.6) [18], similarly to the median OS of the present study (7.5 months, range 2-45).

On the contrary, the prospective SARC (Sarcoma Alliance for Research Through Collaboration) study exploring the activity of GD in a group of different bone sarcomas (including Ewing, chondrosarcoma and 14 patients with osteosarcoma), demonstrated a lack of activity in all cohorts, including osteosarcoma [15].

In our series, grade 3-4 neutropenia or thrombocytopenia was observed in $25 \%$ of the patients, with no difference according to gemcitabine dose and with only 1

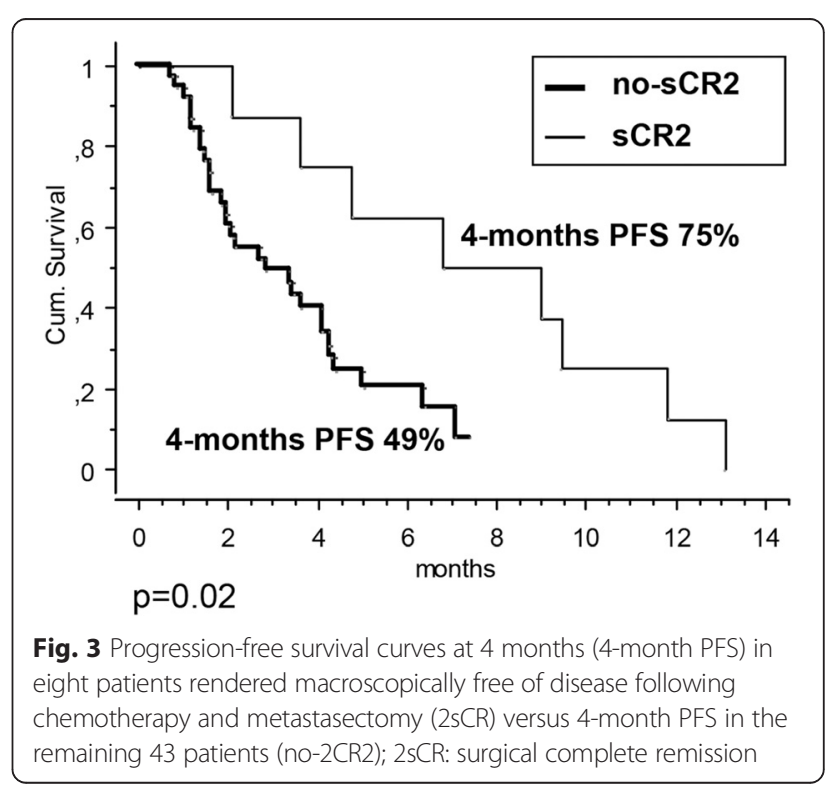


patient with febrile neutropenia, which was described in about $14 \%$ of cycles after HDIFO [24]. More importantly our study confirms that taxane-related hypersensitivity reactions (HSR) can be an issue: 3 (6\%) patients experienced allergic reactions during docetaxel infusion (two following the first cycle and one after 4 cycles), and were subsequently treated with gemcitabine monotherapy; in the other 2 pediatric cases, grade 3 Stephen Johnson syndrome and capillary leak syndrome were documented, in one patient each (Table 4). Taxane-related HSR usually occurs within the first minutes of infusion in up to $30 \%$ of patients, without premedication, and $\leq 4 \%$ with antihistamine and steroid premedication. This is dose and rate-dependent, and adequate premedication and patient monitoring on commencing treatment is recommended [25].

Compared to other therapeutic options in this setting, we did not observe renal toxicity. This can be an issue with HDIFO in about $25 \%$ of cases, and in half of these, irreversible renal failure can occur [24].

Unlike sorafenib, which can cause pneumothorax and permanent treatment discontinuation in about $3 \%$ of patients [8, 9], gemcitibine and docetaxel-induced tumor-shrinkage, was not associated with pneumothorax in our series."

\section{Conclusion}

In conclusion, there are few new active regimens for patients with relapsed osteosarcoma following multimodality therapy [26]. To our knowledge, this is the largest series of patients treated with GD for relapsed bone sarcoma published. While a prospective SARC phase II study showed marginal activity for GD in metastatic osteosarcoma [15], partly due to limitations in clinical trial design, as suggested by the authors themselves [15], we believe that the combination is active and should be included in the therapeutic armamentarium of metastatic osteosarcoma.

\begin{abstract}
Abbreviations
CTCAE: Common Toxicity Criteria for Adverse Events; D: docetaxel; G: gemcitabine; HGS: high-grade spindle cell sarcoma of bone; OS: overall survival; PD: progressive disease; PFS: progression-free survival; PR: partial response; RECIST: Response Evaluation Criteria In Solid Tumors; sCR2: surgical complete response; SD: stable disease.
\end{abstract}

\section{Competing interests}

The authors declare that they have no competing interests.

\section{Authors' contribution}

EP, RLJ, PP, SF: substantive intellectual contribution to conception and design of study, drafting and revision of manuscript, statistical analyses and interpretation of data $A P, E M, R L J, S P, G G, S F$ : final approval of the version to be published EP, AP, MC, AL, CM, LC, FF, SA, AT: patients selection/inclusion All authors have read and approved the contents of this manuscript and declare that the work is original and has not been submitted or published elsewhere.

\section{Acknowledgments}

This work was supported by: the Associazione Onlus "il Pensatore: Matteo Amitrano"; 5 \% donation (Italy), EU FP7 Eurosarc, Biobanca Oncologia Emilia Romagna.

Dr E. Pamerini's work is supported by the Emilia-Romagna Region HighTechnology Network, PROMETEO Laboratory at Istituto Ortopedico Rizzoli. We thank Cristina Ghinelli for figures and table editing.

\section{Author details}

${ }^{1}$ PROMETEO Laboratory/Chemotherapy Unit, Istituto Ortopedico Rizzoli, Bologna, Italy. ${ }^{2}$ University of Washington/ Fred Hutchinson Cancer Research Center, Seattle, USA. ${ }^{3}$ Oncoematologia Pediatrica INT, Milan, Italy. ${ }^{4} \mathrm{UO}$ Oncoematologia Pediatrica Pisa, Pisa, Italy. ${ }^{5}$ Oncoematologia Pediatrica Torino, Torino, Italy. ${ }^{6}$ RCCS Candiolo, Torino, Italy. ${ }^{7}$ Oncoematologia Azienda Ospedaliera Universitaria Meyer Pediatrica, Firenze, Italy. ${ }^{8}$ Laboratory Research, Istituto Ortopedico Rizzoli, Bologna, Italy. ${ }^{9}$ PROMETEO Laboratory/ Section of Chemotherapy, Research, Innovation \& Technology (RIT)

Department, Istituto Ortopedico Rizzoli, Via Pupilli, 1, 40136 Bologna, Italy.

Received: 17 July 2015 Accepted: 13 April 2016

Published online: 20 April 2016

References

1. Ferrari S, Palmerini E. Adjuvant and neoadjuvant combination chemotherapy for osteogenic sarcoma. Curr Opin Oncol. 2007;19:341-6.

2. Bielack SS, Kempf-Bielack B, Delling G, et al. Prognostic factors in high-grade osteosarcoma of the extremities or trunk: an analysis of 1,702 patients treated on neoadjuvant cooperative osteosarcoma study group protocols. J Clin Oncol. 2002;20:776-90.

3. Ferrari S, Briccoli A, Mercuri M, et al. Pos t relapse survival in osteosarcoma of the extremities: prognostic factors for long-term survival. J Clin Oncol. 2003;21:710-5.

4. ESMO/European Sarcoma Network Working Group. Bone sarcomas: ESMO Clinical Practice Guidelines for diagnosis, treatment and follow-up. Ann Oncol. 2014;25 suppl 3:iii113-23.

5. Rosen G, Forscher C, Lowenbraun S, et al. Synovial sarcoma. Uniform response of metastases to high dose ifosfamide. Cancer. 1994;73:2506-11.

6. Patel SR, Vadhan-Raj S, Papadopolous N, et al. High-dose ifosfamide in bone and soft tissue sarcomas: results of phase II and pilot studies-dose-response and schedule dependence. J Clin Oncol. 1997;15:2378-84.

7. Duffaud F, Egerer G, Ferrari S, et al. A phase II trial of second-line pemetrexed in adults with advanced/metastatic osteosarcoma. Eur J Cancer. 2012:48:564-70.

8. Grignani G, Palmerini E, Dileo P, et al. A phase II trial of sorafenib in relapsed and unresectable high-grade osteosarcoma after failure of standard multimodal therapy: an Italian Sarcoma Group study. Ann Oncol. 2012;23:508-16.

9. Grignani G, Palmerini E, Ferraresi V, Italian Sarcoma Group, et al. Sorafenib and everolimus for patients with unresectable high-grade osteosarcoma progressing after standard treatment: a non-randomised phase 2 clinical trial. Lancet Oncol. 2015:16:98-107.

10. Pautier P, Floquet A, Penel N, et al. Randomized multicenter and stratified phase II study of gemcitabine alone versus gemcitabine and docetaxel in patients with metastatic or relapsed leiomyosarcomas: a Federation Nationale des Centres de Lutte Contre le Cancer (FNCLCC) French Sarcoma Group Study (TAXOGEM study). Oncologist. 2012;17:1213-20.

11. Maki RG, Wathen JK, Patel SR, et al. Randomized phase II study of gemcitabine and docetaxel compared with gemcitabine alone in patients with metastatic soft tissue sarcomas: results of sarcoma alliance for research through collaboration study 002 [corrected]. J Clin Oncol. 2007;25:2755-63.

12. Hensley ML, Maki R, Venkatraman E, et al. Gemcitabine and docetaxel in patients with unresectable leiomyosarcoma: results of a phase II trial. J Clin Oncol. 2002;20:2824-31.

13. Merimsky O, Meller I, Kollender Y, et al. Gemcitabine in bone sarcoma resistant to Doxorubicin-based chemotherapy. Sarcoma. 2000;4:7-10.

14. Navid F, Willert JR, McCarville MB, et al. Combination of gemcitabine and docetaxel in the treatment of children and young adults with refractory bone sarcoma. Cancer. 2008;113:419-25.

15. Fox E, Patel $\mathrm{S}$, Wathen JK, et al. Phase II study of sequential gemcitabine followed by docetaxel for recurrent Ewing sarcoma, osteosarcoma, or unresectable or locally recurrent chondrosarcoma: results of Sarcoma 
Alliance for Research Through Collaboration Study 003. Oncologist. 2012;17:321.

16. Gosiengfiao Y, Reichek J, Woodman J, et al. Gemcitabine with or without docetaxel and resection for recurrent osteosarcoma: the experience at Children's Memorial Hospital. J Pediatr Hematol Oncol. 2012;34:e63-5.

17. He AN, Tang LN, Shen Z, et al. Efficacy and safety of gemcitabine-docetaxel combination therapy for recurrent or refractory high-grade osteosarcoma in China: a retrospective study of 18 patients. Jpn J Clin Oncol. 2012;42:427-31.

18. Song BS, Seo J, Kim DH, et al. Gemcitabine and docetaxel for the treatment of children and adolescents with recurrent or refractory osteosarcoma: Korea Cancer Center Hospital experience. Pediatr Blood Cancer. 2014;61: 1376-81.

19. Van Glabbeke M, Verweij J, Judson I, EORTC Soft Tissue and BoneSarcoma Group, et al. Progression-free rate as the principal end-point for phase II trials insoft-tissue sarcomas. Eur J Cancer. 2002;38:543-9.

20. Harris MB, Cantor AB, Goorin AM, et al. Treatment of osteosarcoma with ifosfamide: comparison of response in pediatric patients with recurrent disease versus patients previously untreated: a PediatricOncology Group study. Med Pediatr Oncol. 1995;24(2):87-92.

21. Lee SH, Chang MH, Baek KK, et al. High-dose ifosfamide as second- or third-line chemotherapy in refractory bone and soft tissue sarcoma patients. Oncology. 2011;80(3-4):257-61.

22. Berger M, Grignani G, Ferrari S, et al. Phase 2 trial of two courses of cyclophosphamide and etoposide for relapsed high-risk osteosarcoma patients. Cancer. 2009;115:2980-7.

23. Rodríguez-Galindo C, Daw NC, Kaste SC, et al. Treatment of refractory osteosarcoma with fractionated cyclophosphamide and etoposide. J Pediatr Hematol Oncol. 2002;24:250-5.

24. Berrak SG, Pearson M, Berberoğlu S, Ilhan IE, Jaffe N. High-dose ifosfamide in relapsed pediatric osteosarcoma: therapeutic effects and renal toxicit. Pediatr Blood Cancer. 2005;44(3):215-9.

25. Joerger M. Prevention and handling of acute allergic and infusion reactions in oncology. Ann Oncol. 2012;23 Suppl 10:x313.

26. Benjamin RS. Osteosarcoma: better treatment through better trial design. Lancet Oncol. 2015:16:12-3.

\section{Submit your next manuscript to BioMed Central and we will help you at every step:}

- We accept pre-submission inquiries

- Our selector tool helps you to find the most relevant journal

- We provide round the clock customer support

- Convenient online submission

- Thorough peer review

- Inclusion in PubMed and all major indexing services

- Maximum visibility for your research

Submit your manuscript at wuw biomedcentral.com/submit 